\title{
JPWL - an Extension of JPEG 2000 for Wireless Imaging
}

\author{
Frédéric Dufaux \\ Ecole Polytechnique Fédérale de \\ Lausanne (EPFL) \\ CH-1015 Lausanne, Switzerland \\ Frederic.Dufaux@epfl.ch
}

\author{
Giuseppe Baruffa, Fabrizio Frescura \\ Università degli Studi di Perugia \\ IT-06125 Perugia, Italy \\ baruffa@diei.unipg.it, \\ frescura@diei.unipg.it
}

\author{
Didier Nicholson \\ Thales Communications \\ F-92704 Colombes, France \\ Didier.Nicholson@fr.thalesgroup.com
}

\begin{abstract}
In this paper, we present an overview of the JPWL standardization activity. JPWL is an extension of JPEG 2000 for the efficient transmission of JPEG 2000 images over an error-prone wireless network. More specifically, JPWL supports a set of tools for error protection and correction, including Forward Error Correcting codes (FEC), Unequal Error Protection (UEP), data partitioning and interleaving.
\end{abstract}

\section{INTRODUCTION}

The transmission of image and video content over wireless networks is becoming ubiquitous. Wireless networks are characterized by the frequent occurrence of transmission errors, which put strong constraints on the transmission of digital imagery. Given its high compression efficiency, JPEG 2000 is a very appealing solution in wireless multimedia applications.

For these reasons, JPEG launched in 2002 a new activity referred to as Wireless JPEG 2000 or JPWL [1][2]. JPWL is currently a Final Draft International Standard and is expected to become an International Standard in 2006. In this paper, we give an overview of JPWL and present two use cases.

The goal of JPWL is to extend the baseline specification in order to allow for the efficient transmission of JPEG 2000 image data over an error-prone wireless transmission environment. More specifically, JPWL supports tools and methods to protect the codestream against transmission errors, such as the ones in [3][4][5]. It also defines means to describe the sensitivity of the codestream to transmission errors, and to describe the locations in the codestream of residual transmission errors. JPWL is notably addressing the protection of the image header, Forward Error Correcting (FEC) codes, Unequal Error Protection (UEP), data partitioning and interleaving.

JPWL is not linked to a specific network or transport protocol, but provides a generic solution for the robust transmission of JPEG 2000 codestreams over error-prone networks. While the main target of JPWL is wireless applications, the same tools can also be employed in other error-prone applications.

\section{JPWL OVERVIEW}

The main functionalities of the JPWL system are: to protect the codestream against transmission errors, to describe the degree of sensitivity of different parts of the codestream to transmission errors, and to describe the locations of residual errors in the codestream.

An example of a JPWL system is illustrated in Figure 1. At the transmission side, a JPWL encoder consists of three modules running concurrently: a JPEG 2000 baseline encoder compressing the input image, a generator of the error sensitivity description, and a processor applying the error protection tool. The result is a JPWL codestream robust to transmission errors. At the receiving side, a JPWL decoder is also composed of three modules: a processor for correcting errors, a generator of the residual errors description and a JPEG 2000 baseline decoder.

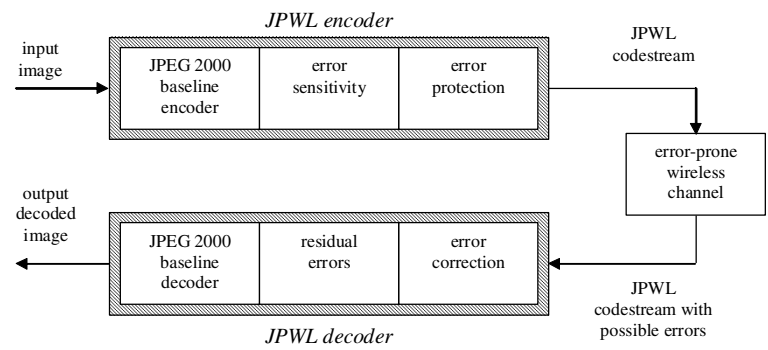

Figure 1. JPWL system description: encoder and decoder.

The JPWL syntax defines four new marker segments: Error Protection Capability (EPC), Error Protection Block (EPB), Error Sensitivity Descriptor (ESD), and Residual Error Descriptor (RED). They are described in more details hereafter.

\section{A. Error Protection Capability (EPC)}

The EPC marker segment indicates which JPWL normative and informative tools are used in the codestream. More specifically, EPC signals whether the three other normative marker segments defined by JPWL, namely ESD, 
RED, and EPB, are present in the codestream. Its syntax is illustrated in Figure 2.

Furthermore, EPC signals the use of informative tools which have been previously registered with the JPWL Registration Authority (RA). Upon registration, each tool is assigned an ID, which uniquely identifies it. These informative tools allow for error resilience and/or error correction, and include techniques such as error resilient entropy coding, FEC codes, UEP, data partitioning and interleaving. EPC may also contain parameters relative to these informative tools. Therefore, this syntax allows for a flexible use of existing tools and the rollout of new ones in the future.

\begin{tabular}{|c|c|c|c|c|}
\hline \multirow[t]{3}{*}{ EPC } & $L_{E P P}$ & $P_{C A C}$ & $\mathrm{DL}$ & $P_{E P C}$ \\
\hline & $I^{(1)}$ & $\operatorname{Lil}_{10}^{(1)}$ & & \\
\hline & $I D^{(<)}$ & $\mathrm{L}_{10}(z)$ & & \\
\hline$\ldots$ & $I D^{(n)}$ & $\mathrm{L}_{10}(\mathrm{n})$ & & \\
\hline
\end{tabular}

Figure 2. Syntax for the EPC marker segment.

EPC is a unique marker and $\mathrm{L}_{\mathrm{EPC}}$ is the length of the marker segment. $\mathrm{P}_{\mathrm{CRC}}$ are parity check bits to verify that the EPC marker segment is not corrupted. DL indicates the total data length the EPC marker segment is referring to. $\mathrm{P}_{\mathrm{EPC}}$ signals the presence of the ESD, RED and EPB marker segments, and the use of informative techniques in the codestream. ID $^{(i)}$ is the identification number issued by the RA for tool $\mathrm{i}, \mathrm{P}_{\mathrm{ID}}{ }^{(\mathrm{i})}$ contains parameters for tool $\mathrm{i}$, and $\mathrm{L}_{\mathrm{ID}}{ }^{(\mathrm{i})}$ is the length of $\mathrm{L}_{\mathrm{ID}}{ }^{(\mathrm{i})}+\mathrm{P}_{\mathrm{ID}}{ }^{(\mathrm{i})}$ in bytes.

\section{B. Error Protection Block (EPB)}

The primary function of EPB is to protect the Main and Tile-part headers. However, it can also be used to protect the remaining of the bitstream. The EPB marker segment contains information about the error protection parameters and redundancy data used to protect the codestream against errors [3]. Its syntax is shown in Figure 3.

\begin{tabular}{|c|c|c|c|c|}
\hline$E P B$ & $L_{E P B}$ & $D_{E P B}$ & ${ }^{L}$ & $P_{E P B}$ \\
\hline
\end{tabular}

Figure 3. Syntax for the EPB marker segment.

EPB is a unique marker and $\mathrm{L}_{\mathrm{EPB}}$ is the length of the marker segment. $\mathrm{D}_{\mathrm{EPB}}$ specifies the usage of EPB. $\mathrm{LDP}_{\mathrm{EPB}}$ is the length of the data to be protected by the redundant information carried within the current EPB marker segment. $\mathrm{P}_{\mathrm{EPB}}$ allows changing the error correcting code used in the remaining data. Finally, EPB Data contains the data to perform error correction, typically redundancy bits.

\section{Error Sensitivity Descriptor (ESD)}

The ESD marker segment contains information about the sensitivity of codestream to errors. This information can be exploited when applying UEP techniques. Straightforwardly, more powerful codes are used to protect the most sensitive portion of the codestream. This information can also be used for selective retransmissions as proposed in [5]. More specifically, a larger number of retransmissions are attempted for the most critical parts of the codestream. Finally, the information carried in ESD could also be used for other non-JPWL applications such as efficient rate transcoding or smart prefetching. Its syntax is given in Figure 4.

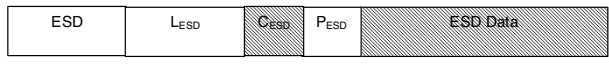

Figure 4. Syntax for the ESD marker segment.

ESD is a unique marker and $\mathrm{L}_{\mathrm{ESD}}$ is the length of the marker segment. $\mathrm{C}_{\mathrm{ESD}}$ specifies which component the ESD data is referring to. $\mathrm{P}_{\mathrm{ESD}}$ describes the usage of ESD. For instance, ESD can use a byte-range mode, a packet mode or a packet-range mode. Also, error sensitivity data can be expressed in different ways, such as relative values, Mean Square Errors (MSE) or Peak-Signal-Noise-Ratio (PSNR). Finally, ESD Data contains the record of error sensitivity data itself.

\section{Residual Error Descriptor (RED)}

The RED marker segment signals the presence of residual errors in the codestream. Indeed, a JPWL decoder may fail to correct all the errors in a codestream. RED allows signaling the location of such residual errors. This information can then be exploited by a JPEG 2000 decoder in order to better cope with errors. For instance, the decoder could request retransmission, conceal the errors or discard the corrupted information. Its syntax is given in Figure 5.

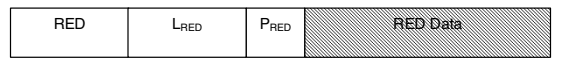

Figure 5. Syntax for the RED marker segment.

RED is a unique marker and $\mathrm{L}_{\mathrm{RED}}$ is the length of the marker segment. $P_{\text {RED }}$ specifies the usage of RED. In particular, RED can use a byte-range mode, a packet mode or a packet-range mode. Finally, RED Data contains the record of residual error data itself.

\section{CASE STUDY: EPB FOR UNEQUAL ERROR PROTECTION}

In this first case study, we consider the use of EPB to apply UEP on a JPEG 2000 codestream. For instance, it is possible to take advantage of the ESD information in order to select the most appropriate technique for protecting different parts of the codestream. UEP can be applied in two ways, either inside the codestream thanks to the flexible structure of EPB, or by separating the codestream in different parts, each of them being protected differently and sent to different error prone environments. 
The following example explains how to realize UEP with EPBs. The Main and Tile-part headers can be protected using EPBs. Additional EPBs are inserted in the Tile-part header to protect data packets. It is important to note that the resulting protected stream still complies with JPEG 2000 syntax. EPB parameters are protected using one of the default error correction codes, while the data to be corrected uses the code described in the EPB parameters set.

As an application, let us consider the image 'Woman' with three different layers for an overall rate of $0.5 \mathrm{bpp}$. The application of UEP is illustrated in Figure 6.

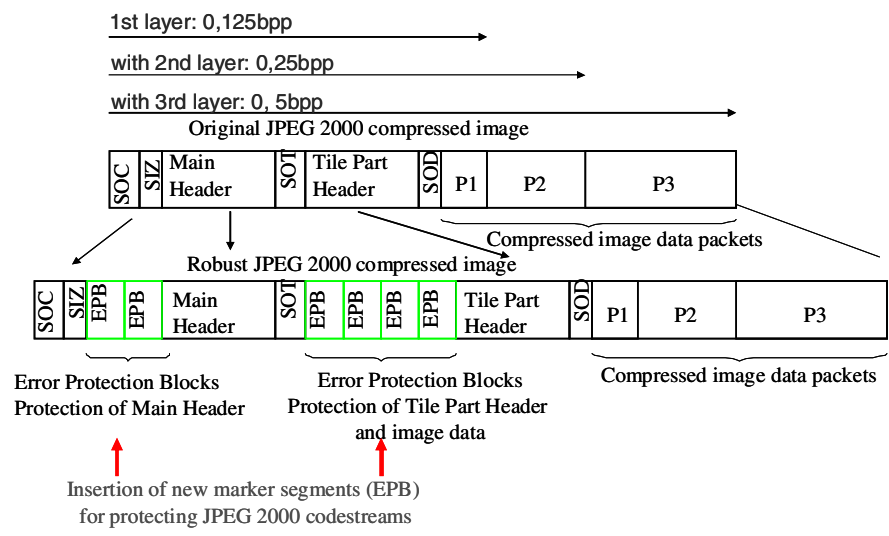

Figure 6. Layers and UEP for test image 'Woman'.

Applying an overall protection rate $\mathrm{R}=2 / 3$ for both Equal Error Protection (EEP) and UEP with Reed Solomon (RS) codes over a Binary Symmetric Channel (BSC) channel for 500 trials gives the results presented in Figure 7. RS $(30,20)$ was used for the three layers of EEP case and $\operatorname{RS}(50,20)$, $\operatorname{RS}(30,20)$ and no protection were respectively used for the UEP case. These error correction codes not being part of the JPWL predefined codes, they have to be indicated in the EPC descriptor.

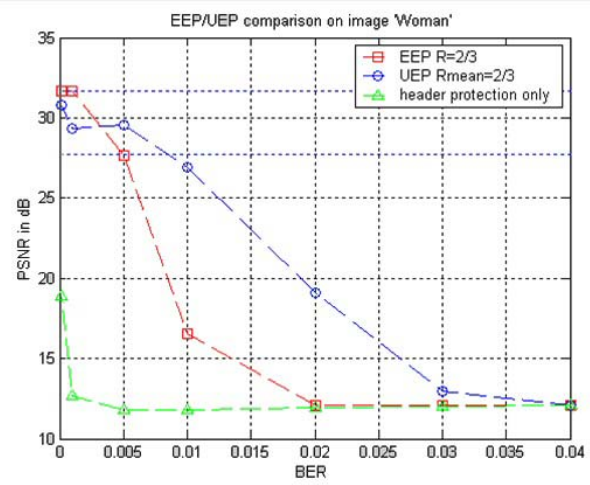

Figure 7. PSNR results for EEP and UEP on 'Woman'.

It can be observed that as soon as the Bit Error Rate (BER) of the channel is above the EEP code capacity, EEP performance quickly degrades, whereas for UEP the stronger protection level provided by upper layer code allows for an acceptable PSNR up to BER $=10^{-2}$.
Visual results at $\mathrm{BER}=10^{-2}$ are proposed in Figure 8. It illustrates the dramatic impact of bit errors for EEP $(\mathrm{PSNR}=18.83 \mathrm{~dB})$ when compared to UEP $(\mathrm{PSNR}=26.95 \mathrm{~dB})$.
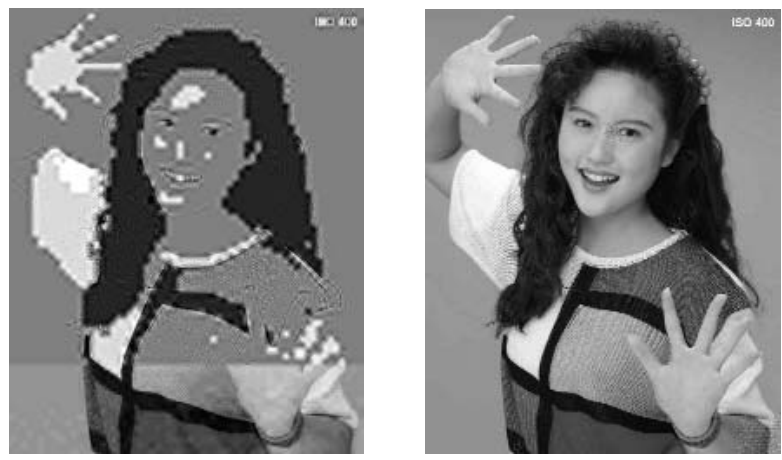

Figure 8. Visual results for EEP and UEP on 'Woman' at BER $=10^{-2}$.

\section{CASE STUDY: INTERLEAVING FOR TRANSMISSION OVER WLAN}

In this second case study, we study the use of data interleaving for the transmission of JPEG 2000 codestreams over Wireless LAN (WLAN).

The transmission characteristics of the WLAN environment generally require a strong protection method to be adopted on the transmitted codestreams. Burst errors tend to appear on the received signal. From the point of view of overlying protocols, this results in a probability of packet loss which can be quite high and variable in time. It is well known that data interleaving associated with channel coding is an optimum strategy for protecting video transmission over nonstationary channels [4]. Since data interleaving can only be applied before channel coding, and the syntax of the codestream has to be preserved, a symmetric de-interleaving strategy must be applied before the transmission. When we apply this concept to the data protection strategy used in JPWL, it is equivalent to the computation of the parity bytes of RS codes employing codewords which are not formed by consecutive data, but rather result from a periodic sampling of the codestream [6]. Moreover, depending on the codestream size and channel characteristics, it may be more convenient to interleave among a set of consecutive codestreams, as illustrated in Figure 9. In order to assess the performance of this method, a reference 2048x1080 (2K) video sequence has been compressed according to the Digital Cinema (DC) specifications [7], and prepared in Motion JPEG 2000 format for transmission. The compressed video sequence has then been JPWL-encoded using the basic RS $(37,32)$ error correction code.

In a first set of simulations, we adopted a Gilbert-Elliot (GE) discrete channel model, characterized by the presence of error bursts. Figure 10(a) shows the achieved PSNR performance for an interleaving depth of a single frame (intra-frame interleaving). As expected, the performance gain with respect to the absence of interleaving is evident. The 
same figure also shows the achieved performance for an interleaving depth of 2, 6 and 10 frames (inter-frame interleaving). As shown, the interleaving performance is substantially identical when different interleaving depths are observed. This behavior can be explained considering that the interleaving depth is already large enough to prevent the error bursts from exceeding the error correction capability of the adopted RS code, also in the single frame case.

In a second set of simulations, we adopted a different model for the transmission channel. It consists in partitioning the compressed video sequences into packets of fixed length, with a certain probability of packet loss. If a packet is lost, it is replaced by a random sequence of bytes, thus simulating the behavior of a transmission protocol (such as, for example, UDP) over a noisy discrete channel. Figure 10(b) shows the obtained results. With a packet length of up to $64 \mathrm{kB}$, the performance of the interleaved configurations is basically the same; there is, however, a difference with respect to a packet length of $100 \mathrm{kB}$. This value does not represent a typical case, because some of the most common protocols do not allow for such a large dimension of packets (e.g., the maximum length for a UDP packet is $64 \mathrm{kB}$ ).

\section{ACKNOWLEDGMENT}

EPFL and Thales Communications contributions to this work are partially supported by the European Project WCAM http://www.ist-wcam.org (IST Contract 507204) funded under the European Commission IST $6^{\text {th }}$ Framework Program. The authors would like to thank all the individuals who participated in the development of JPWL.

\section{REFERENCES}

[1] "JPEG 2000 image coding system - Part 11: Wireless JPEG 2000 Final Draft International Standard", ISO/IEC JTC1/SC29/WG1 WG1N3819, Nov. 2005

[2] F. Dufaux and D. Nicholson, "JPWL: JPEG 2000 for wireless applications", in SPIE Proc. Applications of Digital Image Processing XXVII, Denver, CO, Aug. 2004.

[3] D. Nicholson, C. Lamy-Bergot, X. Naturel, C. Poulliat, "JPEG 2000 backward compatible error protection with Reed-Solomon codes," IEEE Trans. Consumer Electron., vol. 49, pp. 855-860, Nov. 2003.

[4] F. Frescura, M. Giorni, C. Feci, and S. Cacopardi, "JPEG2000 and MJPEG2000 transmission in 802.11 wireless local area networks," IEEE Trans. Consumer Electron., vol. 49, pp. 861-871, Nov. 2003.

[5] M. Grangetto, E. Magli and G. Olmo, "Error sensitivity data structures and retransmission strategies for robust JPEG 2000 wireless imaging", IEEE Trans. on Consumer Electronics, vol. 49, no. 4, pp. 872-882, Nov. 2003

[6] P. G. Sherwood and K. Zeger, "Error protection for progressive image transmission over memoryless and fading channels," IEEE Trans. on Commun., vol. 46, pp. 1555-1559, Dec. 1998.

[7] Digital Cinema Initiatives, LLC, "Digital Cinema System Specification V5.1 (draft a0331),” April 2005.

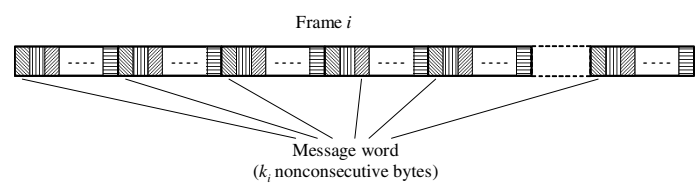

(a)

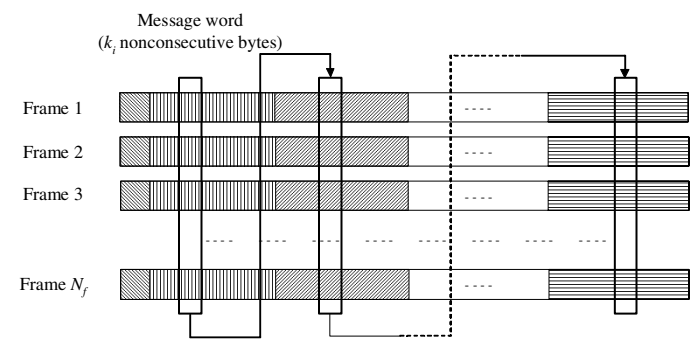

(b)

Figure 9. (a) Intra-frame mode: RS parity bytes are computed for a message word of $k_{i}$ nonconsecutive data bytes, i.e. interleaved data bytes.

(b) Inter-frame mode: parity bytes are computed for a message word composed of bytes interspersed within $N_{f}$ frames. Note how, in both cases, the syntax of the JPEG2000 codestream is not modified and fully complies with the standard.

(a)

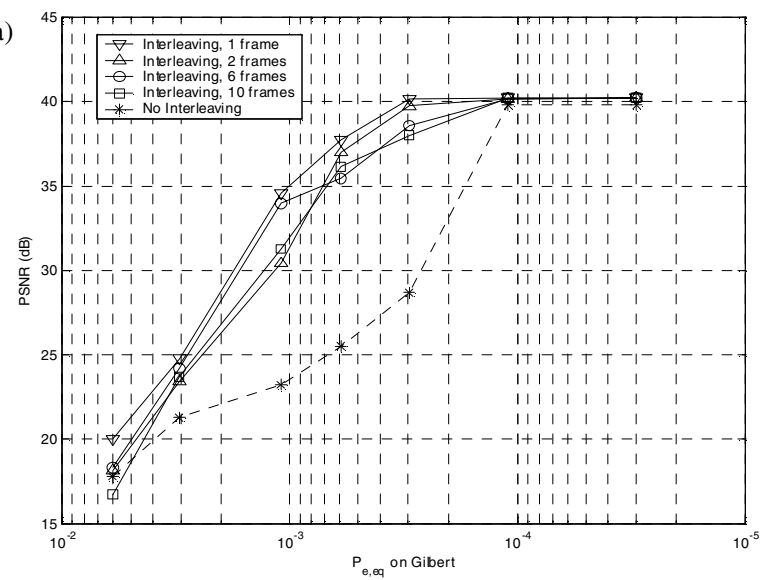

(b)

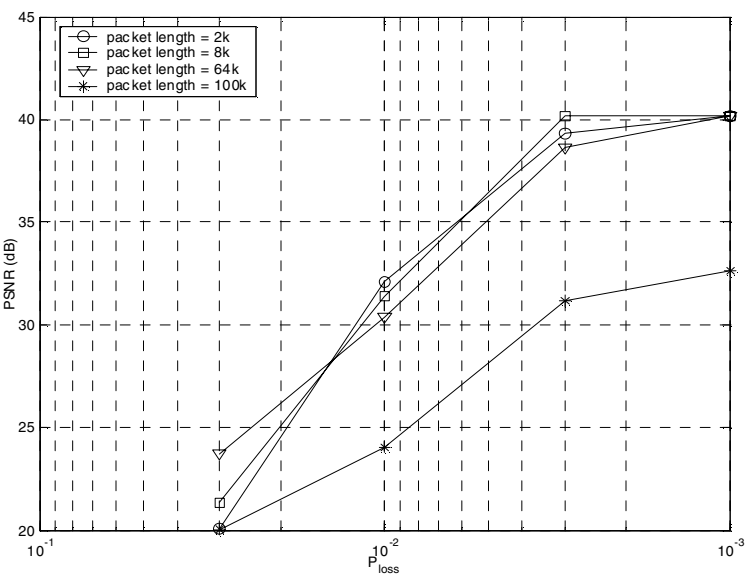

Figure 10. Average PSNR performance for transmission over a packet-loss channel. (a) Results obtained for different values of the interleaving depth, plotted in terms of the equivalent BER of the GE channel. (b) Results obtained for different protocol packet lengths (the interleaving is performed over a single frame), plotted in terms of the packet loss probability. 